\title{
A Literature Review on Communication Levels to Develop Language-Based Augmentative and Alternative Communication Diagnostic Classification System in Korea
}

\author{
Sangeun Shin', HyunJu Park² \\ 'Department of Speech-Language Pathology, Chungnam National University, Daejeon, Korea \\ 2Division of Speech-Language Pathology, Gachon University, Seongnam, Korea
}

\author{
Received: November 21, 2020 \\ Revised: December 21, 2020 \\ Accepted: December 21, 2020 \\ Correspondence: \\ HyunJu Park, PhD \\ Division of Speech-Language \\ Pathology, Gachon University, \\ 1342 Seongnam-daero, Sujeong-gu, \\ Seongnam 13120, Korea \\ Tel: +82-31-750-8881 \\ Fax: +82-31-750-8837 \\ E-mail: phj8747@gachon.ac.kr
}

\begin{abstract}
The purpose of this study was to develop a language-based augmentative and alternative communication (AAC) diagnostic classification system (LAAC-D) that can be used in the assessment and intervention phases of AAC for people with complex communication needs in Korea. A comprehensive literature review was conducted focusing on linguistic competence and the four major factors (intentionality, symbolic use, word combination, and grammatical complexity) that play essential roles in classifying communication and expressive language development stages. Seven pieces of literature reported communication levels that are being used for AAC diagnostic system. Among them, only one reflected all factors into the communication level. Most of the literature did not consider the grammatical complexity factor. After examining the characteristics and limitations of each literature, the LAAC-D was developed consolidating the evidence and reflecting the four factors. The characteristics and clinical implications of LAAC-D were discussed.
\end{abstract}

Key Words: Augmentative and alternative communication, Communication levels, Diagnosis, Linguistic competence.

\section{INTRODUCTION}

복합적인 의사소통 요구를 지닌 사람에게 보완대체의사소통 (augmentative and alternative communication, AAC)을 통해 효과적인 중재를 제공하기 위해서는 평가단계에서 이들의 의사 소통에 영향을 미치는 감각, 운동, 인지, 언어능력에 대한 평가 와 함께 $\mathrm{AAC}$ 도구 사용 능력에 대한 세부 평가가 종합적으로 실시되어야 한다(Beukelman \& Mirenda, 2013). 일반적으로 언 어병리학의 평가(assessment) 과정에 진단 단계(diagnosis phase)가 수반되는 것과 같이(Darley, 1991; Peterson \& Marquardt, 1994), 그 한 분야인 AAC 또한 평가 후 정확한 진단을 내리는 것이 대상자의 현재 의사소통 수준을 파악하고, 현재와 미래에 요구되는 의사소통 능력을 갖추도록 하는 데 중요한 나 침반 역할을 하게 된다(Dodd \& Gorey, 2014). 아울러 진단은 대상자의 의사소통 수준이 계획한 $\mathrm{AAC}$ 중재를 통해 어느 정 도 향상되었는가를 중재 전-후의 비교를 통해 알려주는 지표

(c) This is an Open Access article distributed under the terms of the Creative Commons Attribution Non-Commercial License (https://creativecommons.org/licenses/by-nc/4.0) which permits unrestricted non-commercial use, distribution, and reproduction in any medium, provided the original work is properly cited.
로도 활용될 수 있기에 임상 현장에서 차지하는 그 중요성이 작지 않다.

\section{$\mathrm{AAC}$ 의사소통 능력과 언어적 능력의 관계}

$\mathrm{AAC}$ 대상자의 의사소통 능력(communicative competence) 을 적절히 파악하기 위해서는 그 능력에 영향을 미치는 4가지 영역, 즉 언어적 능력(linguistic competence), 조작적 능력(operational competence), 사회적 능력(social competence), 전략 적 능력(strategic competence)과 함께 다양한 사회심리학적 요 인과 환경적 요인에 대한 고려가 필요하다(Light, 1989, 2003). 그중에서도 언어적 능력은 대상자의 수용 및 표현 언어 기술뿐 아니라 그들이 속한 공동체 언어에 대한 지식을 갖추고 상징 (예: 몸동작, 기호, 글자, 그림 등)을 사용하여 의미적, 구문적으 로 표현해 낼 수 있는 능력을 말하는데, 의사소통 능력을 결정 짓는 데 있어 기본적이면서도 중추적인 역할을 담당한다 (Blockberger \& Sutton, 2003; Light \& McNaughton, 2014). 여기서 말하는 상징(symbol)이란 지시 대상(referent)을 나타 내거나 뜻하는 것으로(Vanderheiden \& Yoder, 1986), 사람들 간에 메시지를 주고 받는 과정에서 사용된다(Beukelman \& 
Mirenda, 2013). AAC 대상자는 대부분 주요 의사소통 방식 (modality)인 말(speech) 사용에 어려움을 보이기에 이를 보완 하고 대체할 수 있는 상징 사용을 학습하게 된다(Mineo Mollica, 2003). 그리고 이들이 말 대신 사용할 수 있는 상징에는 그 림, 사진과 같이 2 차원으로 표상(representation)되거나 실물, 미니어처, 사물의 일부분과 같이 3 차원으로 표상된 도구적 상 징(aided symbols)과 자신의 신체만을 사용하여 표현하는 수 어(sign language)나 몸짓(gesture) 등의 비도구적 상징(unaided symbols)이라는 두 유형이 존재한다.

문해력이 아직 발달되지 않았거나 후천적인 이유로 그 능력 이 손상된 AAC 대상자는 시각적 의사소통 방식인 그림 기반 의 언어 표상(picture-based language representation)에 주로 의존하게 된다(Stephenson \& Linfoot, 1996). 일반적으로, 생 후 6개월 정도면 친숙한 사람과 사물을 2차원적 이미지와 연결 할 수 있는 능력이 어느 정도 생기기에 그림 기반의 언어 표상 방법은 나이가 어리거나, 처음 언어 개념을 익힐 때, 또는 인지 발달이 아직 진행 중이거나 인지장애를 동반하고 있는 아동을 대상으로 적용하기에 적절한 것으로 알려져 있다(DeLoache et al., 1979; Mineo Mollica, 2003). 반면에 그림 기반의 언어 표상 방식은 사회구성원이 주로 사용하는 의사소통 산출 방식인 말 (speech)과 그 성격이 다를 뿐만 아니라(Smith \& Grove, 2003), $\mathrm{AAC}$ 대상자 입장에서는 입력되는 방식(예: 말)과 산출되는 방 식(예: 그림상징)이 서로 불일치한다는 문제가 발생한다. 이로 인해 입력과 산출 방식이 일치된 조건에 있는 일반 아동에 비 해 AAC 대상자는 표현 언어의 발달 및 습득에 어려움을 보일 수 있다(Smith \& Grove, 1999). 언어장애를 지닌 경우에도 대 부분의 아동은 언어발달의 시기가 늦어질 뿐, 전형적인 발달 과정을 따르는 것으로 알려져 있다(Fowler, 1998; Rondal, 1994). 따라서 효과적인 중재 계획과 이행을 위해서는 진단 시 이들의 의사소통 수준이 전반적인 발달 과정의 어느 단계에 속 하는지를 정확하게 파악할 필요가 있다.

\section{의사소통 및 표현 언어 발달의 주요 요인}

일반적인 의사소통 발달 단계를 살펴보면, 먼저 전의도적 (preintentional)인 '초보적 의사소통 행동(primitive communicative behaviors)'과 '목표지향적 의사소통 행동(perlocutionary communicative behaviors)'을 거쳐 생후 8개월 이후부 터는 의도성(intentionality)이 있는 '도구적 전환기 행동(transitional/instrumental communicative behaviors)'을 보이기 시 작한다. 이후 몸동작, 발성 패턴, 억양 그리고 기능적인 초어 (proto-words)를 사용하여 분명하게 자신의 의도를 표현하려 는 '언어 이전 의사소통 행동(illocutionary communicative behaviors)'을 보이게 되고 대략 14 개월 이후에는 주로 말을 사
용하여 '언어적 의사소통 행동(locutionary communicative behaviors'을 보이게 된다. 이때부터는 의미적, 문법적으로 표 현 언어 능력이 빠른 속도로 발달하게 되는데, 단단어 수준의 어휘 사용이 16 24개월에 어휘 폭발(vocabulary burst)을 거치 면서 단어 조합을 보이게 되고, 구문 발달기로 접어들면서는 문 법 규칙을 적용해 다양한 문장구조를 만들 수 있게 된다 (Bates, 1974; Bates et al., 1975; Owens, 2015; Wetherby \& Prizant, 1989). Kim(2003)은 한국 아동이 보이는 구문 발달을 2 3세를 기준으로 단순한 구문 구조를 사용하는 '기본 문법 탐 색기'와 한 문장에 여러 주어와 서술어가 들어간 문장 구조를 산출할 수 있는 '기본 문법 세련기', 그리고 5세 이후에는 다양 한 구문 구조와 문법형태소를 사용하여 복잡한 복문도 세련되 게 사용하는 '고급 문법기'로 구분하기도 하였다.

의사소통 및 표현 언어 발달 단계를 구분하는 주요 요인을 살펴보면, 1) 의사소통의 의도성(intentionality), 2) 구어 사용, 3) 단어 조합성(단단어 vs. 단어 조합)과 4) 문법적 복잡성이 작 용하고 있음을 알 수 있다. AAC 대상자는 일반적인 의사소통 및 언어 발달 단계에서 보이는 구어 대신 그림, 사진, 몸짓, 수 어 등의 상징을 통한 의사소통(symbolic communication)을 보인다는 점을 제외하고 다른 요인들이 동일하게 작용하면서 언어가 발달하게 된다(Brady et al., 2012). 이때 AAC 대상자의 상징 사용은 다중방식(multi-modality) 의사소통을 지향하는 AAC의 특성을 고려하여(Beukelman \& Mirenda, 2013; Hanson et al., 2013) 도구적 상징(예: 그림, 사진, 실물, 글자)을 비도 구적 상징(예: 발성, 몸짓, 수어, 얼굴표정)과 위계적으로 구분하 기보다는 다양한 상징이 동시에 효과적으로 사용될 수 있음을 주지할 필요가 있다.

\section{국내 AAC 진단분류}

그렇다면 국내에서 활용하고 있는 $\mathrm{AAC}$ 진단분류에는 위와 같은 주요 발달 요인들이 어느정도 반영되어 있을까? 현재 특 수교육과 언어 재활 분야에서 널리 사용되고 있는 $\mathrm{AAC}$ 진단 분류체계는 한국보완대체의사소통평가(Korean AAC Assessment, KAA) (Kim et al., 2016)에 수록되어 있는 '의사소통 단 계'이다. '의사소통 단계'는 총 3단계로 구성되어 있는데 첫 번째 단계인 '전의도적 의사소통자'는 아직 의사소통 행동을 의도적 으로 사용하지 못하는 단계로, 표정 변화나 발성을 보이기도 하나 아직까지는 자신의 행동이 의사소통 상대자에게 영향을 미칠 수 있다는 것을 알지 못한다고 정의되어 있다. 두 번째 단 계인 ‘목표지향적/비도구적 상징 의사소통자’는 자신의 행동이 의사소통 상대자에게 영향을 미칠 수 있다는 것을 인식하나 1 단계와 마찬가지로 표정, 발성, 시선 등의 비도구적 행동을 통 해 표현하는 단계로 설명되고 있다. 마지막으로 세 번째 단계인 
'도구적 상징 의사소통자'는 2단계와 마찬가지로 자신의 행동이 의사소통 상대자에게 영향을 미칠 수 있다는 것을 인식하며, 2단계와는 달리 그림, 글자, 말, 물체와 같은 구체적 또는 추상 적 상징을 통해 표현할 수 있다고 보고 있다. $\mathrm{KAA}$ 에서 정의하 는 각 단계를 그 내용을 기준으로 살펴보면 앞서 기술한 의사 소통 및 표현 언어 발달 단계를 구분하는 4 가지 요인 중 의사 소통의 의도성 여부를 기준으로 1 단계를 2,3 단계와 구분하고 있다. 비록 2단계 명칭에 포함된 '목표지향적'이라는 용어 때문 에 Bates(1974)의 의사소통 발달단계에서의 전의도적인 '목표지 향적 의사소통 행동에 부합되는 것으로 볼 수 있겠으나, 본 연 구에서는 명칭보다 Kim et al.(2016)이 기술한 2단계 내용에 보 다 초점을 맞춰 분석하기로 한다.

$\mathrm{KAA}$ 는 상징의 도구성 여부에 따라 비도구적 상징을 사용 하는 2단계와 도구적 상징을 사용하는 3단계를 위계적으로 구 분하는 특징을 보인다. AAC 대상자가 말 대신 상징을 사용하 기에 도구적/비도구적 상징을 사용하여 언어를 표현하는 것은 언어적 의사소통 행동을 결정짓는 주요한 요인으로 작용할 것 이다. 그러나 상징 사용을 도구성에 따라 위계적으로 구분하고 있기 때문에 다중방식으로 의사소통하는 $\mathrm{AAC}$ 대상자에게 적 용할 때에는 제한적일 수 있다. 예를 들어, 비도구적 상징 유형 인 몸짓과 수어를 통해 2 3개의 낱말을 조합하여 표현할 수 있 는 $\mathrm{AAC}$ 대상자와 그림 상징을 사용하여 몇몇 단단어 수준의 표현을 보이는 $\mathrm{AAC}$ 대상자를 평가할 경우, 도구적 상징을 사 용했다는 이유로 전자는 2단계에, 후자는 3단계에 속하게 된다.

또한 $\mathrm{KAA}$ 는 의사소통의 의도성과 상징 사용 여부를 고려하 고는 있으나 단어 조합성과 문법적 복잡성은 포함하고 있지 않 아 '언어적 의사소통 행동' 단계에 있는 $\mathrm{AAC}$ 대상자의 의미적, 형태·구문적 발달수준을 파악하는 데 어려움이 있다. 가령 그 림 상징을 통해 제한된 단단어 수준의 표현을 보였던 대상자가 체계적인 $\mathrm{AAC}$ 중재를 통해 그림과 글자 상징을 조합하여 복 잡한 문장까지도 표현할 수 있게 된다 하더라도 그 대상자는 초기 평가와 재평가 모두에서 최고 단계인 의사소통 3단계의 진단을 받게 된다. 진단보고서에는 대상자의 향상된 언어적 능 력을 부연 설명할 수 있겠으나 진단분류에서 명확하게 그 수준 을 확인해 줄 수 있다면 현재의 언어 수준뿐만 아니라 중재를 통한 향상된 수준을 비교하는 데에도 보다 용이할 것이다.

의사소통 의도성과 상징 사용 여부, 특히 도구적 상징을 사용 하는지를 확인할 필요가 있는 $\mathrm{AAC}$ 대상자에게는 $\mathrm{KAA}$ 의 3단 계가 간편한 기준으로 활용될 수 있을 것이다. 그러나 위에서 살 펴본 바와 같이 대상자의 전반적인 $\mathrm{AAC}$ 의사소통 능력에 언어 적 능력(Light, 1989)이 미치는 영향이 크고(Light \& McNaughton, 2014), 이를 위해서는 정확한 표현 언어 수준을 진단하여 중재하는 것이 중요하므로 의미적·문법적 발달 요인을 고려한
언어 기반의 진단 분류가 마련될 필요가 있다.

이에 본 연구에서는 해외에서 $\mathrm{AAC}$ 대상자에게 사용하고 있 는 진단분류체계를 고찰함으로써 의사소통 및 언어 발달 단계 의 지표를 반영한 진단분류체계가 있는지를 살펴보고, 이들 분 류체계의 특징과 제한점을 고려하여 국내 $\mathrm{AAC}$ 대상자에게 적 용할 수 있는 '언어 기반 진단분류(language-based AAC diagnostic classification, LAAC-D)'를 제안하고자 하였다. 그리 고 이를 위한 연구 문제는 다음과 같다.

첫째, 해외 문헌에 소개된 $\mathrm{AAC}$ 진단분류는 의사소통 및 표 현 언어 발달의 주요 요인(의도성, 상징 사용성, 단어 조합성, 문 법적 복잡성)을 어느 정도 반영하고 있는가?

둘째, 각각의 진단분류가 갖고 있는 특징과 제한점은 무엇인가? 셋째, 해외 진단분류를 통합 및 보완하고 의사소통 및 표현 언어 발달 단계의 주요 요인이 모두 반영된 언어 기반 AAC 진 단분류체계는 무엇인가?

\section{MATERIALS AND METHODS}

\section{문헌 검색}

본 연구에서는 1990년 1월부터 2020년 10월까지 국내외 학 술지와 단행본으로 출간된 문헌자료를 검색하였다. 검색에 사 용된 데이터베이스는 ERIC과 ProQuest였고, 키워드는 AAC (또는 augmentative and alternative communication)와 함께 expressive language (또는 communication) skills, communication profiles (또는 scales, levels), communicative complexity로 하였다. 검색 결과 총 455개의 연구를 확인하였고, 이 중 전체 문서를 열람할 수 있는 102 개에 대해 51 개의 연구가 중 복되어 제외되었다.

\section{연구 절차}

51 개 연구에 대해 문헌 선정 기준을 바탕으로 검토하면서, 본문 내에 $\mathrm{AAC}$ 를 필요로 하는 대상자에게 적용할 수 있는 또 다른 의사소통 수준이 소개된 경우, 동일한 선정 기준을 적용 하여 추가적으로 포함여부를 결정하였다. 그 결과 11 개의 추가 논문이 분석에 포함되었다.

포함 조건(inclusion criteria)으로는 1) 의사소통 수준이 $\mathrm{AAC}$ 를 필요로 하는 장애군(예: 자폐범주성장애, 지적장애, 뇌 성마비)에게 적용할 수 있도록 고안된 경우, 2) 의사소통 수준 을 분류할 때 상징 사용을 포함한 경우, 3) 진단분류의 임상적 활용도를 높이기 위해 의사소통 수준이 적어도 3 개 이상 위계 적으로 제시된 경우로 하였다. 배제 조건(exclusion criteria)으 로는 1) 언어의 사회성 또는 화용적 측면이 강조된 경우, 이는 Light(1989)의 분류에서 언어적 능력이 아닌 사회적 능력에서 
설명되는 바, 언어적 능력을 중심으로 살펴보고자 하는 본 연구 의 목적과 다소 거리가 있으므로 배제하기로 하였다. 또한, 2) 수 용 언어 수준이 표현 언어 수준과 하나의 진단분류에 혼재되어 제시된 경우에는 두 언어능력이 보이는 상호작용이 본 연구의 핵심적인 관심 주제와 거리가 있기에 분석에서 배제하였다. 마 지막으로 3) 한 문헌에서 언어적 능력 외에 다른 $\mathrm{AAC}$ 의사소 통 능력(예: 조작적 능력, 전략적 능력)을 함께 다룬 경우에는 본 연구의 목적을 고려하여 언어적 능력 외에 다른 영역은 분 석에서 제외하였다.

각각의 문헌이 선정 기준을 모두 충족하는지의 여부는 본 연 구의 저자 2 인이 독립적으로 코딩 처리(부합하는 경우 $=1$, 그렇 지 않은 경우 $=0)$ 한 후 연구자 간 일치도를 살펴보았으며, 총 7 개의 문헌 중 6 개의 문헌에 대해 일치(85.71\%)를 보이는 것으로 나타났다. 불일치한 1 개 문헌에 대해서는 검토 과정에서 누락된 것으로 확인되어 포함하기로 결정하였다. 최종 문헌 7개를 선정 하기까지 진행된 절차가 요약적으로 Figure 1에 제시되어 있다.

최종 선정된 문헌은 1) '의사소통 복잡성 척도(Communication Complexity Scale, CCS)' (Brady et al., 2012), 2) ‘의사소통 매트릭스(Communication Matrix)' (Rowland, 2016), 3) 'AAC 프로파일(AAC profile)' (Kovach, 2009) 중 언어적 능력 수준 (level of the linguistic competence) 부분, 4) '표현 의사소통 기 술 프로파일(profiles of the expressive communication skills)' (Mar \& Sall, 1999a, 1999b) 중 의사소통의 복잡성(communicative complexity)과 의도성(intentionality) 부분, 5) '트리플 C (The Triple C)' (Bloomberg et al., 2009), 6) '의사소통 능력 수준(levels of communicative abilities)' (Visvader, 2013), 그리고 7) '독립성 수준(levels of independence)' (Dowden, 1999)이다.

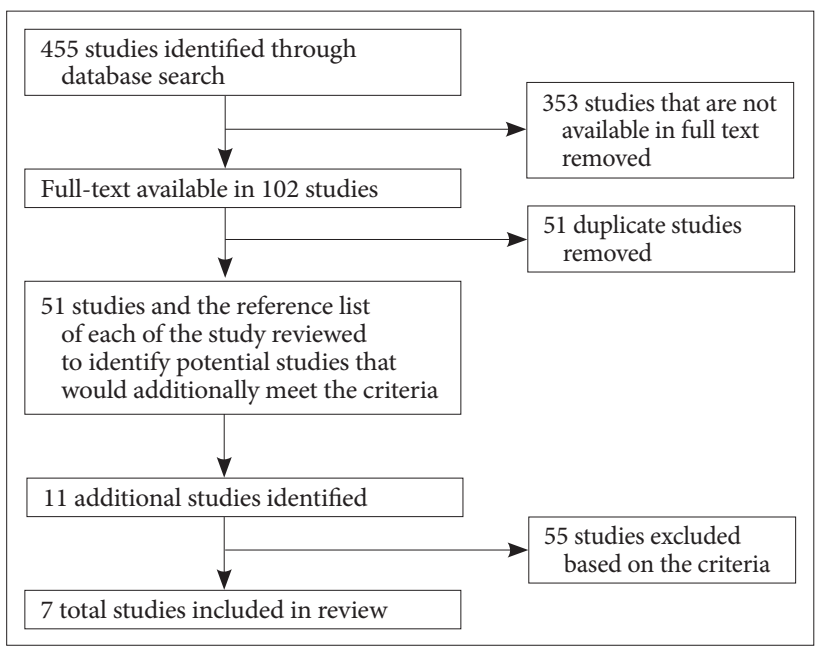

Figure 1. Flowchart of searching process and results used in the study.

\section{자료 분석 방법}

첫 번째 연구 질문과 관련하여 각 문헌의 분류가 의사소통 및 표현 언어 발달 단계에서 확인된 주요 요인과 일치하는가 여 부를 살펴보기 위해 1) 의도성, 2) 상징 사용성, 3) 단어 조합성, 4) 문법적 복잡성별로 반영 여부를 분석하였다. 그 외의 요인에 대해서는 기타로 분류하여 살펴보았다. 본 연구에서는 요인별 질적분석방법을 사용하였는데, 이는 Chadwick et al.(2019)이 중도지적장애와 중도중복장애 아동 대상의 진단평가도구들이 갖는 근거 기반 정도를 판별하기 위해 항목별로 응답자의 반응 을 체크리스트 형태로 분석한 것을 참고한 것이다.

두 번째 연구 질문에 대해서는 각 문헌의 진단분류가 의사소 통 및 표현 언어의 발달 수준을 구분하고 있는 특징과 제한점 을 상세히 분석기술하는 방식으로 진행하였다. 특히, $\mathrm{KAA}$ 의 진단분류에서 충족되지 않는 의사소통 발달(Bates, 1974)의 마 지막 단계인 '언어적 의사소통 단계'에 초점을 맞추고, 단어 조 합성과 문법적 복잡성 요인에 따라 단계가 세밀하게 구분되어 있는지를 살펴보았다.

마지막으로, 의사소통 및 표현 언어 발달 단계의 주요 요인들 을 모두 반영한 언어 기반 $\mathrm{AAC}$ 진단분류체계를 확립하기 위 해 4 개의 주요 요인에 따라 문헌들을 질적으로 통합 분석한 결 과를 제시하였다.

\section{RESULTS}

\section{$\mathrm{AAC}$ 대상자를 위한 의사소통 수준의 분류 요인}

각 문헌에서 의사소통 수준을 분류하는 기준으로 작용한 요 인을 Table 1에 제시하였다. 전체적으로 확인된 요인은 총 11개 로, 1) 의도성(intentionality), 2) 상징 사용(symbol use), 3) 단 어 조합성(word combination), 4) 문법적 복잡성(grammatical complexity)이 주요 의사소통 발달 단계의 분류 요인으로 작용 하였으며, 그 외에 5) 지남력(orientation), 6) 잠재적 의사소통 행동(potentially communicative behavior, PCB), 7) 전형성 (conventionality), 8) 의사소통 기능(communication function), 9) 상징 어휘의 구체성(concrete/abstract symbols), 10)의 사소통의 능동성(active/passive), 11) 독립성(independency) 등 이 포함되었다.

각각의 요인별로 살펴보면, 의도성이 반영된 것은 '의사소통 복잡성 척도(Brady et al., 2012), '의사소통 매트릭스(Rowland, 2016), '표현 의사소통 기술 프로파일(Mar \& Sall, 1999a, 1999b)', '트리플 C(Bloomberg et al., 2009)' 등 총 4개였다. 상징 사용은 본 연구의 선정 조건이었던 만큼 모든 문헌에서 고려되었다. 반 면, 언어적 의사소통 단계의 중요 요인인 단어 조합성과 문법적 복잡성은 각각 5 개와 2 개의 문헌에서 보고되었다. 특히, 문법적 
Table 1. Factors to classify the communication levels

\begin{tabular}{|c|c|c|c|c|c|c|}
\hline \multirow[b]{2}{*}{ Title of the communication level } & \multirow[b]{2}{*}{ Study } & \multicolumn{5}{|c|}{ Factors on the communication level } \\
\hline & & Intentionality & $\begin{array}{c}\text { Symbol } \\
\text { use }\end{array}$ & $\begin{array}{c}\text { Word } \\
\text { combination }\end{array}$ & $\begin{array}{l}\text { Grammatical } \\
\text { complexity }\end{array}$ & Others \\
\hline $\begin{array}{l}\text { Communication Complexity Scale } \\
\text { (CCS) }\end{array}$ & $\begin{array}{l}\text { Brady et al. } \\
(2012)\end{array}$ & $\checkmark$ & $\checkmark$ & $\checkmark$ & & Orientation, independency \\
\hline Communication Matrix & Rowland (2016) & $\checkmark$ & $\checkmark$ & $\checkmark$ & & $\begin{array}{l}\text { Conventionality, } \\
\text { communication function, } \\
\text { concrete/abstract symbols, } \\
\text { independency }\end{array}$ \\
\hline $\begin{array}{l}\text { AAC profile: } \\
\text { Level of the linguistic competence }\end{array}$ & Kovach (2009) & & $\checkmark$ & $\checkmark$ & $\checkmark$ & \\
\hline $\begin{array}{l}\text { Profiles of the expressive } \\
\text { communication skills: } \\
\text { communicative complexity \& } \\
\text { intentionality }\end{array}$ & $\begin{array}{l}\text { Mar \& Sall } \\
\quad(1999 a, 1999 b)\end{array}$ & $\checkmark$ & $\checkmark$ & $\checkmark$ & $\checkmark$ & \\
\hline $\begin{array}{l}\text { The Triple C: } \\
\text { Checklist of communication } \\
\text { competencies, revised }\end{array}$ & $\begin{array}{l}\text { Bloomberg et al. } \\
\text { (2009) }\end{array}$ & $\checkmark$ & $\checkmark$ & & & Active/passive \\
\hline Levels of communicative abilities & Visvader (2013) & & $\checkmark$ & $\checkmark$ & & \\
\hline Levels of independence & Dowden (1999) & & $\checkmark$ & & & Independency \\
\hline
\end{tabular}

복잡성은 4개 요인 중 가장 적게 반영된 것으로 나타났다. 두 요인을 모두 포함하지 않은 문헌은 Bloomberg et al.(2009)의 '트리플 C'와 Dowden(1999)의 '독립성 수준'이었다.

\section{해외 진단분류의 특징 및 제한점}

\section{의사소통 복잡성 척도(CCS)}

Table 2에 제시된 바와 같이 '의사소통 복잡성 척도(CCS) (Brady et al., 2012)는 크게 전의도적(preintentional), 의도적 비상징(intentional nonsymbolic) 그리고 의도적 상징(intentional symbolic) 수준으로 대분류하고 있다. 다른 문헌과 차별 화된 $\mathrm{CCS}$ 의 특징은 상징을 사용하기 전 단계인 전의도적 수준 과 의도적 비상징 수준에서 지남력과 잠재적 의사소통 행동 (PCB)의 수준에 따라 10 개의 세부 분류를 적용하고 있다는 점 이다. 여기서 지남력은 주변의 사람, 상황 또는 사물을 인지하 여 그쪽으로 시선을 옮기거나 몸의 방향을 바꾸는 행위 등을 말한다. $\mathrm{PCB}$ 는 의사소통을 시도하는 것처럼 보이는 잠재적 의 도성이 담긴 행동을 말하는 것으로 몸동작, 시선, 발성 등이 이 에 해당한다.

$\mathrm{CCS}$ 를 개발한 Brady et al.(2012)은 상징 사용 이전의 의사 소통 행동이 그 이후의 언어발달 단계에서 구어나 다른 상징을 사용하여 의미적, 문법적으로 확장하는 과정을 예측하기 때문 에 세밀한 분류체계를 갖췄다고 밝히고 있다. 또한, 상징 사용 이 아직까지는 제한적인 대상자를 위해 서열척도화된 분류체
계의 필요성을 강조하고 있다. 가령 지남력과 $\mathrm{PCB}$ 의 요인에 따 라 달라지는 점수 체계를 살펴보면, 전의도적 의사소통 단계에서 는 무반응(no response $)=0$ 점, 각성(alerting) $=1$ 점, 사람, 상황, 또는 사물 하나에 대한 단일 지남력(single orientation) = 2점, 단일 지남력 + 잠재적 의사소통 행동 $(\mathrm{PCB}) 1$ 개 = 3점, 단일 지남력 +1 개 이상의 $\mathrm{PCB}=4$ 점, 2 개 대상에 대한 지남력(dual orientation $)=5$ 점을 포함한다. 의도적 비상징 의사소통 단계에 서는 3 개 대상에 대한 지남력(triadic orientation)을 보일 경우 6점, 2 개 대상에 대한 지남력 $+\mathrm{PCB} 1$ 개 = 7점, 2 개 대상에 대 한 지남력 $+\mathrm{PCB}$ 2개 이상 = 8점, 3 개 대상에 대한 지남력 + $\mathrm{PCB} 1$ 개 $=$ 9점, 3 개 대상에 대한 지남력 +1 개 이상의 $\mathrm{PCB}=10$ 점으로 점수를 매기게 된다. 각 단계별 자세한 예와 설명은 Brady et al.(2012)에서 찾아볼 수 있다.

마지막 대분류인 의도적 상징 수준에서는 '단단어 수준의 발 화, 수어, 또는 $\mathrm{AAC}$ 상징 선택(one-word verbalization, sign, or AAC symbol selection)' 단계와 '단어 조합을 통한 발화, 수 어 또는 $\mathrm{AAC}$ 상징 선택(multi-word verbalization, sign, or AAC symbol selection)' 단계를 포함하고 있다. 의도적 상징 수준에서의 두 단계는 단어 조합성을 고려하고는 있으나 문법 적 복잡성은 특별히 고려되어 있지 않다는 제한점이 있다.

\section{의사소통 매트릭스(Communication Matrix)}

'의사소통 매트릭스(Rowland, 2016)'는 총 7단계의 의사소통 수준을 소개하고 있는데, 여기에 의사소통 독립성과 의사소통 
Language-Based AAC Diagnostic Classification

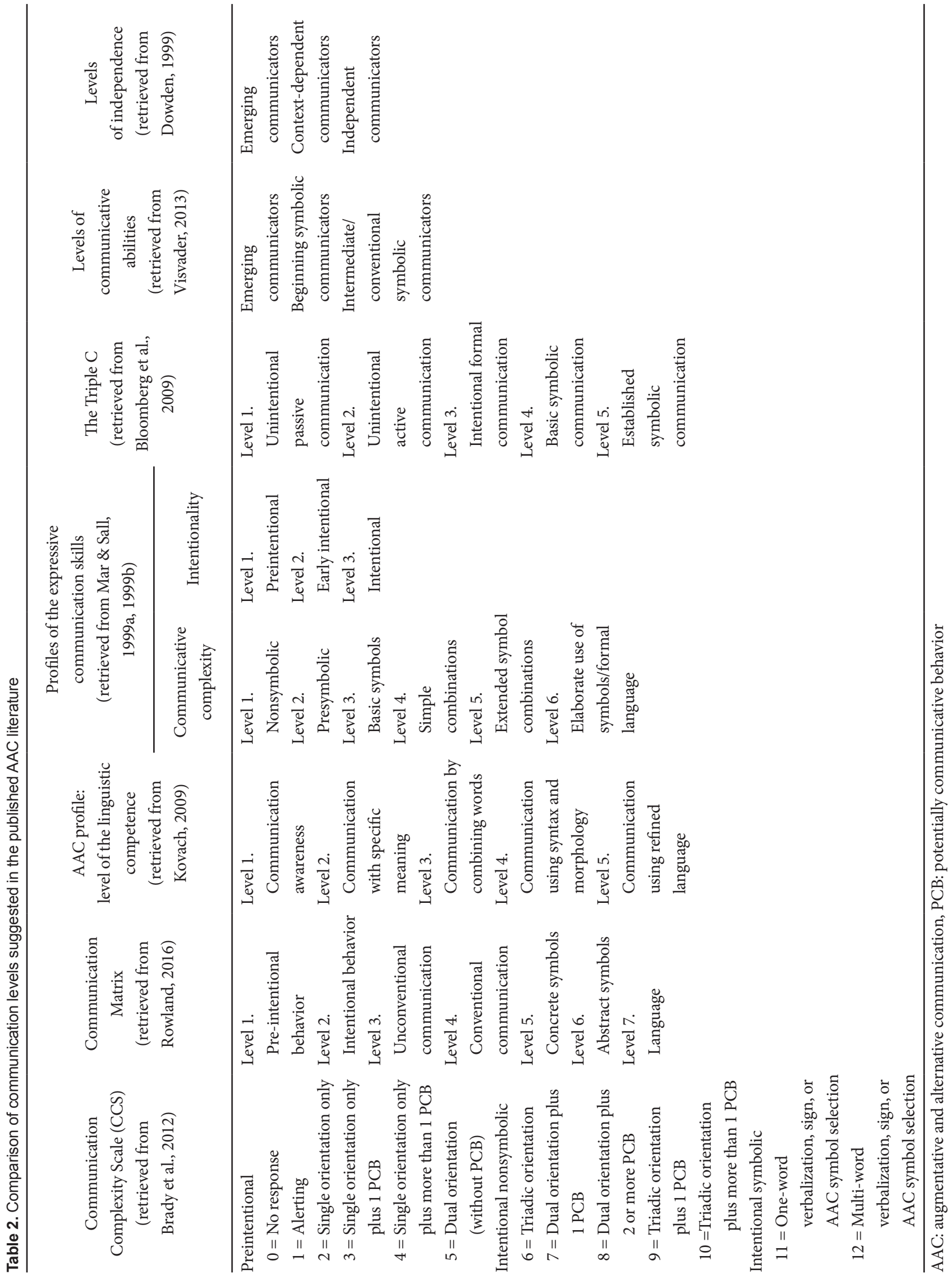


기능(거절, 요구, 사회적 기능, 정보교환)이라는 두 가지 추가적 인 요인을 각 수준별로 확인할 수 있도록 하고 있다. 구체적으 로 살펴보면, 1단계는 전의도적 행동(preintentional behavior) 을 말하며, 2단계 의도적 행동(intentional behavior), 3단계 비 전형적 의사소통(unconventional communication), 4단계 전형 적 의사소통(conventional communication), 5단계 구체적 상 징(concrete symbols), 6단계 추상적 상징(abstract symbols) 그 리고 마지막 7단계는 언어(language)로 이들 단계가 위계적 층 위를 이루고 있다. 또한 의도성에 따라 1 단계와 2 단계가 구분되 고 단어 조합성에 따라 5,6 단계와 7단계가 구분되나, 문법적 복잡성 요인에 따라서는 단계가 구분되어 있지 않기 때문에 $\mathrm{CCS}$ 와 마찬가지로 대상자가 다양한 의미관계의 조합을 보이다 가 문법적인 발달을 보이는 시점에서 어떠한 세부 발달을 보이 는지는 확인하기 어렵다.

의사소통 매트릭스는 다른 문헌에서 찾아볼 수 없는 전형성 이 고려되어 있어서, 비전형적인 의사소통 행동이 점차 사라지 거나 전형적인 행동 패턴으로 변화하는 과정을 반영하고 있다 는 점이 특징적이다. 또한, 상징의 구체성을 위계적인 단계 안에 포함하여 구체적 상징을 추상적 상징보다 선행된 단계로 보고 있다. 그런데 이러한 분류 단계는 신중히 고려할 필요가 있다. 일례로 임상현장에서는 구체적인 상징을 대상자가 쉽게 인식할 수 있다는 편의성 때문에 먼저 훈련 목표로 삼기도 하는데, 이 는 아동의 실제 어휘 발달에 관한 문헌 내용과 다소 상반될 수 있다. 이미 많은 $\mathrm{AAC}$ 연구에서 연령과 상관없이 구체적 어휘 보다 추상적 어휘가 핵심어휘로 빈도 높게 출현하고 있다는 것 을 일관성 있게 보고하면서 핵심 어휘에 대한 교육과 임상적 중 요성을 강조하고 있기 때문이다(Banajee et al., 2003; Beukelman et al., 1989; Marvin et al., 1994; Shin \& Park, 2020). 가 령, 유아가 일상생활에서 공통적으로 자주 사용하는 고빈도의 핵심어휘(Banajee et al., 2003)에는 I, no, yes, want, it, that, my, you, more, go, help, a, all done 등과 같이 구체적인 어휘 보다는 추상적인 어휘들이 대부분을 차지하며 이러한 어휘를 실물을 가리키거나 사진 및 그림과 같이 지시 대상이 구체화된 상징을 통해서 표현하기란 쉽지 않을 수 있다. 따라서 근거에 기반하였을 때, 일상에서 활용도가 높은 추상적인 핵심어휘 상 징 어휘(예: yes, no, want, more)가 구체적인 상징 어휘(예: cookie)보다 중재의 우선순위에서 밀려야 한다는 주장에 대해 서는 타당성을 확보하기가 쉽지 않아 보인다. 이러한 점에서 의 사소통 매트릭스의 상징 속성에 대한 위계는 자칫 실물이나 그 림 및 사진을 통해 구체적인 상징을 먼저 발달시켜야 하는 것처 럼 잘못 인식될 소지가 있으므로 주의가 필요해 보인다.

\section{AAC 프로파일(AAC profile)}

Kovach(2009)의 AAC 프로파일은 Light(1989)의 언어적 능 력, 조작적 능력, 사회적 능력, 전략적 능력별로 평가를 진행할 수 있도록 고안되었다. 본 연구의 목적상 언어적 능력에서 소개 된 의사소통 수준만을 살펴보면, 1 단계부터 5단계까지, 의사소 통을 인식하는 단계(communication awareness), 특정한 의미 로 의사소통하는 단계(communication with specific meaning), 단어를 조합하여 의사소통하는 단계(communication by combining words), 구문과 형태론적으로 의사소통하는 단계 (communication using syntax and morphology), 그리고 정제 된 언어로 의사소통하는 단계(communication using refined language)를 포함하여 위계적으로 구성되어 있다. 상징 사용의 요인에 따라 1 단계와 나머지 단계가 구분되며, 단어 조합성에 따라 2 단계와 3 단계가 구분된다. 문법적 복잡성은 3 단계와 4단 계를 구분하며, 5 단계는 의미적, 문법적으로 보다 정교한 언어 를 산출하는 단계를 말하므로 두 요인이 복합되어 반영된 것으 로 볼 수 있다. 그러나 $\mathrm{AAC}$ 프로파일은 1단계에서 상징을 사 용하지 않을 뿐 의도성 있는 의사소통 행동을 포함하는 것으 로 해석되므로 전의도적 의사소통 단계(Bates, 1974)를 확실하 게 구분하고 있지 않다는 제한점이 있다.

\section{표현 의사소통 기술 프로파일(Profiles of the expressive} communication skills)

Mar \& Sall(1999a, 1999b)의 '표현 의사소통 기술 프로파일' 은 크게 4개 영역에서 의사소통 수준을 분류하고 있다. 여기에 는 의도성(intentionality) 영역, 의사소통의 복잡성(communicative complexity) 영역, 순서교환하기(turn taking) 영역, 개시 하기(initiating) 영역이 포함되어 있다. 순서교환하기와 개시하 기는 Light(1989)의 AAC 의사소통 능력에서 사회적 능력에 보 다 부합하는 내용이므로 본 연구에서는 의도성 영역과 의사소 통 복잡성 영역만을 살펴보았다. 우선 의사소통 복잡성을 살펴 보면, 1단계 비상징(nonsymbolic), 2단계 전상징(presymbolic), 3단계 기초적 상징(basic symbols), 4단계 단순 상징조합(simple combinations), 5단계 확장된 상징 조합(extended symbol combinations), 마지막으로 6단계 정교한 상징 사용(또는 공식 적 언어의 사용)으로 구성되어 있다. 언어적 복잡성 영역에서의 1단계는 Mar \& Sall(1999a, 1999b)의 의도성 영역에서 전의도 적(preintentional) 단계에 해당하며, 2 단계는 의도성 영역에서 목표지향적 행동이 나타나는 초기 의도적(early intentional) 단 계에 해당하고, 3 6단계는 의도성 영역에서 봤을 때 의도적 (intentional) 단계에 해당한다.

상징 사용의 요인에 따라 1,2 단계와 3 단계 이후가 구분되며, 단어 조합성에 따라 단단어 수준을 보이는 3단계, 2 어 조합인 
4단계와 3 4어 조합을 보이는 5단계가 서로 구분된다. 문법적 복잡성은 5 단계와 6 단계를 구분하는 데 주로 작용하며 둘다 문법규칙이 적용된다는 면에서는 공통되나, 6 단계에서는 복잡 한 문법 및 구문에 따라 언어를 정확하고 유창하게 사용한다 는 점에서 차이가 있다.

다른 문헌과 달리 표현 의사소통 기술 프로파일은 발달 단계 의 주요 지표로 작용하는 4 가지 요인이 모두 반영되었다는 장 점을 지니고 있다. 특히 문법적 복잡성에 따라 구분이 되어 있 어 구문적 언어발달 측면을 위계적으로 판별하고자 할 때 유용 하다.

\section{트리플 C(The Triple C)}

'트리플 C(Bloomberg et al., 2009)'는 의사소통 수준을 총 5 단계, 즉 1단계 비의도적 수동적인 의사소통(unintentional passive communication), 2단계 비의도적 능동적인 의사소통 (unintentional active communication), 상징 사용 이전인 3단 계 의도적 비공식 의사소통(intentional informal communication), 제한된 맥락에서만 상징을 사용하는 4단계 기초적 상 징 의사소통(basic symbolic communication), 다양한 맥락에 걸쳐 상징을 사용하는 5단계 확립된 상징 의사소통(established symbolic communication)으로 구분하고 있다.

다른 문헌에서 제시한 의사소통 수준과 달리 트리플 $C$ 에서 는 비의도적 의사소통 단계를 수동적인 것과 능동적인 것으로 구분하는 특징을 지닌다. 비의도적 수동적인 의사소통이란 내 부 및 외부 자극에 대한 반응으로 생성된 행동이 의사소통 상 대자에 의해 의미가 부여된 것을 말한다. 천천히 움직이는 물체 나 사람을 시각적으로 따라가는 행동을 의사소통 상대자가 알 아채고 그것을 좋아한다 또는 가지고 놀고 싶어한다고 의미부 여하는 경우를 예로 들 수 있다. 반면에 비의도적 능동적인 의 사소통이란 의사소통 상대자에 의해 의미가 부여된 행동이라 는 점은 같으나 자극이 주어져서 반응하는 것이 아니라 자발적 으로 대상에 행동을 개시하는 시도를 보인다는 점에서 차이가 있다. 친숙한 사람에게 다가가는 행위나 여러 사물 중에서 자 신이 원하는 사물을 찾거나 바라보는 행위가 이에 해당한다.

반면에 트리플 $\mathrm{C}$ 는 의사소통 및 표현 언어 발달을 구분하는 주요 요인들 중 의도성과 상징 사용만을 고려하고 있다. 따라서 단어 조합성과 문법적 복잡성에 따른 의사소통 수준을 판별하 고자 할 경우에는 도움이 되지 않는다.

\section{의사소통 능력 수준(Levels of communicative abilities)}

3단계로 구성된 Visvader(2013)의 '의사소통 능력 수준'을 살 펴 보면, 먼저 1단계인 초기 의사소통자(emerging communicators)는 전의도적 단계에서 반사적인 반응(울음, 웃음)을 보이
기도 하고, 비상징적 수단을 사용하여 의도를 가진 의사소통 행 동을 보이는 수준에 있다. 이들의 행동에는 의도성이 없는 경 우와 의도성이 있는 경우가 혼재되어 있을 수 있는데, 아직까지 상징을 사용하여 의사소통하는 데에는 어려움이 있다. 다음으 로 2단계인 초기 상징 의사소통자(beginning symbolic communicators)는 의사소통 의도를 가지고 발성, 몸짓, 수어 등을 통해 간단한 인사말이나 선호하는 활동을 요구할 수 있으며, 원하는 활동을 선택하기 위해 그림이나 사물 기반의 의사소통 전략을 사용할 수도 있다. 또한, 간단한 음성출력장치를 조작하 거나 시도할 수 있다. 그러나 한 번에 하나의 상징을 사용하여 의사소통하는 단계로 상징의 조합이나 구문 능력을 보이지는 않는다. 마지막으로 3단계인 중급/전형적 상징 의사소통자(intermediate/conventional symbolic communicators)는 수어나 그림 상징을 조합하여 간단한 형식의 문장뿐 아니라 복잡한 문 장도 전형적인 구문이나 어순 규칙을 적용하여 표현할 수 있다.

Visvader(2013)가 고안한 '의사소통 능력 수준'은 의도성 요 인을 고려하고는 있으나 이것이 단계를 구분하는 중요 요인으 로 작용하지는 않는다. 또한, 단어 조합성이 2 단계와 3 단계를 구분해 주고는 있으나, 문법적 복잡성 요인이 3단계인 단어 조 합 부분에서 함께 설명되고 있기 때문에 별도의 분류요인으로 역할을 하지는 않는다.

\section{독립성 수준(Levels of independence)}

‘독립성 수준(Dowden, 1999)'에서는 AAC를 통한 의사소통 의 독립적 표현이라는 요인을 적용하여 의사소통 수준을 3 단 계로 나누고 있는데, 초기 의사소통자(emerging communicators), 맥락 의존적 의사소통자(context-dependent communicator), 독립적 의사소통자(independent communicators)가 이 에 해당한다. 초기 의사소통자는 아직 상징을 사용하여 의사 소통하는 데 어려움이 있기에 몸짓, 얼굴 표정, 발성 등을 사용 하여 의사소통한다. 이들은 적절한 $\mathrm{AAC}$ 전략이나 기술을 아 직 사용할 수 없기 때문에 독립성이 가장 낮은 단계에 속한다. 맥락 의존적 의사소통자는 상황에 따라 상징/비상징 방식을 모두 사용하여 안정적으로 의사소통할 수 있으나 자신과 다른 의사소통 방식을 사용하는 사람들의 말을 쉽게 이해하지 못하 기에 타인의 도움을 필요로 할 수 있다. 또한, $\mathrm{AAC}$ 를 사용할 수 있는 능력이 제한적이거나 자신의 $\mathrm{AAC}$ 도구에 어휘가 적절히 탑재되어 있지 않을 경우 새로운 메시지를 생성할 방법이 없으 므로 미리 저장된 어휘 또는 메시지에 제한되어 버리는 맥락 의 존적 상태에 놓이게 된다. 독립적 의사소통자는 어떠한 대화 주 제든지, 대화상대자나 상황의 친숙성에 관계없이 대체적으로 누구의 도움없이 자유롭게 의사소통할 수 있는 사람을 말한다.

한편, Dowden(1999)의 '독립성 수준’에서는 의사소통 및 표 
현 언어 발달의 주요 지표로 고려될 수 있는 의도성, 단어 조합 성 및 문법적 복잡성을 고려하지 않고 있다.

\section{언어 기반 진단분류(LAAC-D) 개발}

의사소통 및 표현 언어 발달의 주요 요인이 모두 반영된 진 단분류를 개발하기 위해 각 요인별로 해외 문헌을 고찰한 결과 Appendix와 같이 LAAC-D가 개발되었다. 각 요인별 근거는 Table 3에 제시되어 있으며 자세한 내용은 다음과 같다.

\section{의도성 및 상징 사용에 대한 근거의 확립}

의도성과 상징 사용은 몇몇 문헌에서 함께 아우르며 살펴보 고 있는 바, 따로 구분하지 않고 함께 근거를 제시하였다. 총 7 개의 문헌 중 의도성과 상징 사용 요인을 모두 포함하고 있는 문헌은 CCS (Brady et al., 2012), 의사소통 매트릭스(Rowland, 2016), 표현 의사소통 기술 프로파일(Mar \& Sall, 1999a, 1999b), 트리플 C(Bloomberg et al., 2009)였다. 이중 LAAC-D 는 $\mathrm{CCS}$, 표현 의사소통 기술 프로파일, 그리고 트리플 $\mathrm{C}$ 를 참 고하여 '전의도적 의사소통', ‘의도적 비상징 의사소통', '의도적 상징 의사소통'의 3단계로 크게 구분하였다.

단계별 용어는 CCS와 동일한 것으로 특별히 일치시킨 이유 는 상징 사용 이전에 나타나는 의사소통 행동이 상징 사용 이 후에 나타나는 언어적 의사소통 발달단계에 긴밀한 영향을 미 치기 때문에(Volterra et al., 2005) 필요에 따라 전의도적 단계 와 비상징 의사소통 단계를 보다 촘촘히 세분화할 필요가 있으 며, 단계 설정의 타당성이 확보되어 있어 국내 연구 및 교육 그 리고 임상에서의 활용가치가 높다고 판단하였기 때문이다.

Mar \& Sall(1999a, 1999b) 또한 이들과 유사하게 전의도적 의사소통 단계에 속하는 비상징 단계를 거쳐 초기 의도적 단계
에서 보이는 전상징 행동을 지나 의도적 단계부터는 상징을 사 용하는 단계로 구분하여 살펴보고 있다. '트리플 C (Bloomberg et al., 2009)'는 '의도적 비상징 의사소통'을 '의도적 비공식 의사 소통'이라고 표현하고 있고 '의도적 상징 의사소통'을 '기초적 상 징 의사소통'과 ‘확립된 상징 의사소통’으로 구분하고 있는데 상징 사용의 여부를 단계의 명칭에서 쉽게 확인하는 것이 어려 워 CCS의 틀을 이용하기로 하였다.

반면에 ‘의사소통 매트릭스'는 의도성에 따라 의사소통 단계를 구분하고는 있으나 의도적 의사소통 단계 내에서 상징 사용을 구체성에 따라 위계적으로 구분하고 있어 $\mathrm{AAC}$ 어휘 연구에 근 거한 임상 적용과 배치되는 제한점을 갖고 있다. 따라서 LAAC$\mathrm{D}$ 의 의도성 및 상징 사용의 직접적 근거에서는 배제하였다.

\section{단어 조합성에 대한 근거의 확립}

7 개의 문헌 중 단어 조합성을 고려하여 의사소통 수준을 분 류하고 있는 것은 총 5개로, CCS (Brady et al., 2012), 의사소통 매트릭스(Rowland, 2016), AAC 프로파일(Kovach, 2009), 표 현 의사소통 기술 프로파일(Mar \& Sall, 1999a, 1999b), 의사 소통 능력 수준(Visvader, 2013)이 근거 확립을 위해 검토되었 다. 전반적으로 단단어 수준과 2어 조합 이상의 단어 조합 수 준으로 구분하고 있는데, '표현 의사소통 기술 프로파일'의 경우 에는 이를 보다 세분화하여 단단어 수준의 '기초적 상징' 단계, 2어 조합의 '단순 상징조합' 단계, 그리고 3 4어 조합의 '확장된 상징 조합'으로 분류하고 있다. 발달 과정상 어휘폭발기를 거쳐 2어 조합이 나타나게 되고, 그 이후 구문 발달이 이루어지는 시 점에서는 3 4어 조합도 자주 관찰되기 때문에 ‘확장된 상징 조합' 단계에서도 문법규칙은 활용된다고 Mar \& Sall(1999a, 1999b)은 기술하였다. 의미적 측면의 발달이 선행되는 점, 그리고 구문 발

Table 3. Evidence on the development of LAAC-D

\begin{tabular}{|c|c|c|}
\hline Factors & Evidence & Levels where evidence was applied in LAAC-D \\
\hline $\begin{array}{l}\text { Intentionality \& } \\
\text { symbol use }\end{array}$ & $\begin{array}{l}\text { - Communication Complexity Scale } \\
\text { (Brady et al., 2012) } \\
\text { - Profiles of the expressive communication skills } \\
\text { (Mar \& Sall, 1999a, 1999b) } \\
\text { - The Triple C (Bloomberg et al., 2009) }\end{array}$ & $\begin{array}{l}\text { I. Preintentional communication } \\
\text { II. Intentional nonsymbolic communication } \\
\text { III. Intentional symbolic communication }\end{array}$ \\
\hline Word combination & $\begin{array}{l}\text { - Communication Complexity Scale } \\
\text { - Communication Matrix (Rowland, 2016) } \\
\text { - AAC profile (Kovach, 2009) } \\
\text { - Profiles of the expressive communication skills } \\
\text { - Levels of communicative abilities (Visvader, 2013) }\end{array}$ & $\begin{array}{l}\text { III-1. Communication with single word } \\
\text { III-2. Communication by combining words }\end{array}$ \\
\hline Grammatical complexity & $\begin{array}{l}\text { - AAC profile } \\
\text { - Profiles of the expressive communication skill }\end{array}$ & $\begin{array}{l}\text { III-3. Communication by combining words using basic } \\
\text { grammatical rules } \\
\text { III-4. Language using more complex grammatical rules }\end{array}$ \\
\hline
\end{tabular}

LAAC-D: language-based AAC diagnostic classification 
달이 집중되는 시기에 3 4어 조합이 많이 출현한다는 점을 고 려하여 본 연구에서는 잉여성을 줄이고 의미 관계의 발달적 측 면에 초점을 맞추어 대다수의 2 분화된 진단분류 기준을 따르 기로 하였다.

따라서 'III-1. 단단어 수준'과 'III-2. 단어 조합 수준'으로 위 계화하고, 이 둘 모두를 'III. 의도적 상징 의사소통' 단계의 하 위 단계로 포함시켜, 언어에 기반을 둔 세부 분류가 필요한 임 상현장에서 활용될 수 있도록 하였다.

\section{문법적 복잡성에 대한 근거의 확립}

문법적 복잡성이 고려된 문헌은 2개로 'AAC 프로파일(Kovach, 2009)'과 '표현 의사소통 기술 프로파일(Mar \& Sall, 1999a, 1999b)'이 이에 해당한다. 'AAC 프로파일'에서는 3단계 인 '단어를 조합하여 의사소통하는 단계'와 4단계인 '구문과 형 태론적으로 의사소통하는 단계'가 형태구문적 활용 여부에 따 라 구분되고 있으며, 마지막 5단계인 '정제된 언어로 의사소통 하는 단계'는 4단계보다 의미적, 구문적으로 발전된 형태의 언 어를 구사하는지에 따라 구분하고 있다. 문법적 복잡성을 문법 규칙의 적용 여부에 따라 3 단계와 4단계로 구분하는 것은 어 느 정도 타당해 보이나, 4단계와 5단계의 구분이 명확하게 기술 되어 있지 않아 어느 정도의 문법적 복잡성이 요구되는 단계인 지 쉽게 파악하기가 어렵다.

Mar \& Sall(1999a, 1999b)의 '표현 의사소통 기술 프로파일' 에서도 얼마나 복잡한 문법과 구문을 활용하는지에 따라 '확 장된 상징 조합' 단계와 마지막 단계인 '정교한 상징 사용(또는 공식적 언어의 사용'을 구분하고 있으나 그 구분점이 명확하지 않은 측면이 있다.

따라서 본 연구에서는 두 문헌에서 확인된 제한점을 고려하 여 단계 명칭을 통해 직관적으로 문법적 복잡성 수준이 반영될 수 있도록 하였다. 이에 'III. 의도적 상징 의사소통' 단계의 하 위 단계로 'III-3. 기본 문법 규칙을 사용한 단어 조합 수준과 'III-4. 복잡한 문법 규칙을 사용한 언어 수준'을 포함하였다. III-3과 III-4의 복잡성 기준은 $\operatorname{Kim}(2003)$ 이 기술한 구문 발 달 단계인 '기본 문법 탐색기', '기본 문법 세련기', '고급 문법기' 를 참고하여 설정하였다. $\operatorname{Kim}(2003)$ 에 따르면 '기본 문법 탐색 기'에는 한국어의 문법 규칙을 적용하여 명사구와 '거'를 중심 으로 한 동사구의 사용을 보인다. 또한 '주어 + 목적어(또는 부 사어) + 서술어' 구조나 ‘주어 + 서술어 + 서술어'와 같은 단순 한 구문 구조를 사용할 수 있다. 또한, 이 단계에서는 주격, 여 격 및 공조격 조사의 사용도 관찰되기 시작하며, 과거 시제를 나타내는 어미의 활용 또한 형태론적 측면에서 관찰할 수 있게 된다. '기본 문법 세련기'에서는 단순한 구문구조에서 벗어나 한 문장 내에 두 개 이상의 주어와 서술어를 사용한다거나 복문
을 사용하는 등 보다 복잡한 문장 구조로 발달하게 되며 문장 의 길이도 길어지게 된다. 또한, 구의 형태에서 절의 형태로 다 양하게 발달하게 되며, 피동과 사동의 표현을 보이기 시작한다. 아울러 격조사 및 인용을 나타내는 관형격 조사의 사용과 다 양한 높임과 시제의 활용 등 문법형태소의 사용 능력이 보다 발달하게 된다. 마지막으로 '고급 문법기' 단계에서는 우리말 문 장의 다양한 구문 구조와 문법형태소를 사용하여 복잡한 복문 도 세련되게 사용할 수 있게 된다.

LAAC-D에서는 Kim(2003)이 설정한 이 기준을 적용함으로 써 한국어를 모국어로 사용하는 $\mathrm{AAC}$ 대상자의 문법 수준이 진단분류를 통해 보다 명확하게 파악될 수 있도록 하였다. 가령 LAAC-D에서의 III-3단계는 문법 규칙을 사용하여 단순한 문 장구조를 보이고, 위에서 언급된 발달상 조기 출현하는 조사와 형태소 활용이 관찰될 수 있다. III-4단계에서는 다양한 조사와 형태소 활용을 통해 좀 더 복잡한 문장(중문, 복문)을 구성할 수 있는 단계이다. 이를 $\operatorname{Kim}(2003)$ 의 3단계 구문 발달 과정과 비교하자면 III-3단계는 '기본 문법 탐색기'에 해당하겠고, III-4 단계는 '기본 문법 세련기' 이후 단계로 이해하면 되겠다.

\section{$\mathrm{LAAC}-\mathrm{D}$ 의 추가 특징에 대한 근거의 확립}

$\mathrm{KAA}$ 와 구분되는 $\mathrm{LAAC}-\mathrm{D}$ 의 차이점이자 III단계 전체에 적용되는 주요한 특징 중 하나는 $\mathrm{AAC}$ 는 다중 방식의 의사소 통을 지향한다는 기본 철학을 고수하여 언어적 개념을 표상한 다면 어떠한 유형의 상징이든 그 유형에 따라 위계화하지 않았 다는 점이다. 여기에서 말하는 상징에는 선행연구에 따라 말 (speech), 수신호(manual signs), 몸짓(gestures) 등의 비도구적 상징과 글자, 그림, 사진, 사물 등의 도구적 상징이 모두 포함되 며, 하나 또는 그 이상을 함께 사용하는 모든 언어적 의사소통 활동을 포괄한다(Brady et al., 2012; Sigafoos et al., 2000).

진단분류에 독립적 수행 수준이 고려되었다는 점도 특징 중 하나이다. AAC 대상자가 보이는 의사소통 수준에서도 독립적 으로 상징을 통해 표현할 수 있는 정도가 다르고 사회적 참여 를 위해서는 독립적인 의사소통이 가능하도록 도와주는 중재 가 요구되는 바(Beukelman \& Mirenda, 2013), 진단 단계에서 이러한 내용이 확인될 필요가 있다. 본 연구에서 고찰한 문헌 중 ‘의사소통 매트릭스'(Rowland, 2016) 또한 이러한 독립성을 진단분류에 포함하고 있다. 독립성의 단계를 구분하는 기준은 특별히 중재와의 연계성을 고려하여 $\operatorname{Dodd(2017)ㅇㅢ~ㅎㅏㄱㅅㅡㅂ~ㅅㅜㅈㅜㄴ~}$ (learning), 출현 수준(emerging), 습득 수준(mastered)을 적용 하였다. 학습 수준이란 목표로 하는 의사소통 행동이 나타나지 않아 상당한 도움(support)을 통해 훈련해야 하는 단계를 말한 다. 출현 수준은 약간의 도움을 주면 목표 행동을 수행할 수 있는 단계를 말하며, 마지막으로 습득 수준은 독립적으로 
$\mathrm{AAC}$ 대상자가 목표로 하는 의사소통 행동을 맥락과 관계없이 표현할 수 있는 단계를 의미한다. 본 연구의 문헌 분석에 포함 된 Dowden(1999)의 '독립성 수준'이 있기는 하나, 여기에는 상 징 사용이라는 요인이 함께 고려되고 있기 때문에 LAAC-D에 서 확립한 의사소통 수준과 내용적인 부분에서 중첩될 소지가 있다고 판단되어 직접적인 근거에서는 배제하였다.

\section{DISCUSSIONS}

$\mathrm{AAC}$ 대상자의 의사소통 수준을 언어발달 단계에 맞추어 이 해하고, $\mathrm{AAC}$ 평가와 중재의 맞물린 과정(Beukelman \& $\mathrm{Mi}^{-}$ renda, 2013; Dodd, 2017)에서 이를 적절히 반영한 교육 및 임 상 서비스를 제공하기 위해서는 다양한 상징을 통한 의사소통 및 표현언어발달 단계가 진단분류에 반영되어 있어야 한다. 특 히 언어적 의사소통 행동 단계에서 대상자가 보이는 의미적, 문 법적 표현 언어의 발달 지표가 현재 널리 사용되고 있는 KAA 에는 반영되어 있지 않은 바, 언어적 능력(Light, 1989)에 대한 보다 세밀한 진단평가를 위해 언어에 기반을 둔 진단분류 체계 가 요구되었다. 이에 본 연구에서는 의사소통 및 표현언어발달 의 주요 요인들을 중심으로 해외 문헌을 고찰하고, 각 문헌에 서 보이는 특징과 제한점을 고려하여 국내 $\mathrm{AAC}$ 대상자에게 활용할 수 있는 언어 기반 진단분류체계를 개발하였다.

$\mathrm{LAAC}-\mathrm{D}$ 은 $\mathrm{KAA}$ 의 강점인 간편한 3 단계 분류체계를 유지 하되 해외 문헌에서 파악된 의도성과 상징 사용 여부를 반영한 대분류 기준을 적용하여(Brady et al., 2012), I단계 '전의도적 의사소통', II단계 ‘의도적 비상징 의사소통', III단계 ‘의도적 상 징 의사소통'으로 크게 구분하였다. 따라서 상징적인 의사소통 단계로 나아가는 대상자들에게 좀 더 면밀한 의사소통 수준을 평가하고자 하는 임상가의 경우에는 Brady et al.(2012)이 제안 한 세부 10 단계를 적용할 수 있도록 하였다. 아울러 $\mathrm{KAA}$ 의 3 단계 분류와 달리 상징의 도구성이 아닌 언어를 표상하는 상 징인지 아닌지에 따라 그 유형에 상관없이 단계가 구분되도록 하였다는 특징을 갖고 있다. 이를 통해 여러 상징 유형을 상황 에 따라 다양하게 사용하는 복합적인 의사소통 요구를 지닌 사람들에게 적용하는 데에도 유용할 것으로 보인다. 또한, Bates(1974)의 의사소통 단계별 명칭과 혼동없이 병용하는 데 에 무리가 없을 것으로 보인다. 즉, $\mathrm{LAAC}-\mathrm{D}$ 의 대분류 3단계를 Bates(1974)의 의사소통 단계와 비교하자면, I단계인 '전의도적 의사소통은 Bates(1974)의 의사소통 발달단계 중 생리적인 상 태를 표현하기 위해 반사적인 행동을 보이는 '초보적 의사소통 행동 단계'와 발성, 울음, 몸짓, 눈맞춤 등을 통해 즉각적인 목 표물 획득을 위해서만 제한된 반응을 하는 목표지향적 의사소 통 행동 단계에 해당한다. II단계인 '의도적 비상징 의사소통'에
서는 의사소통 발달 단계 중 계획된 목적을 이루기 위해 분명 한 발성 및 몸짓 신호를 사용하는 행동을 보이는 '도구적 전환 기 행동' 단계와 초어, 발성 패턴의 변화와 억양 사용, 비정형화 된 몸짓 등을 통해 자신의 의사소통 의도를 보다 명확하게 표 현하려는 '언어 이전의 의도적 의사소통 행동' 단계와 연결될 수 있다. 다음으로 III단계인 ‘의도적 상징 의사소통' 단계는 '언 어적 의사소통 행동 단계'에 해당한다. LAAC-D에서는 이 단 계에서 단어의 조합성과 문법적 복잡성에 따라 다시 하위 단계 가 분류되도록 고안되었기 때문에 III단계의 AAC 대상자 중에 서 표현 언어의 능력을 파악하고자 할 때 이 세부 진단분류가 도움이 될 것으로 보인다.

의사소통 매트릭스와 같이 독립성 수준이 진단분류에 고려 됨으로써, 개인의 표현 언어 수준을 2차원적으로 분석할 수 있 도록 하였다. 이는 평가 시점과 중재 이후의 시점 간에 독립성 이 변화한 수준을 살펴보는 데에도 유용할 것으로 기대한다.

본 연구는 국내 AAC 대상자의 언어적 능력(Light, 1989)에 대한 정확한 진단 평가와 중재를 위해 의사소통 및 표현 언어 의 발달과정을 반영한 언어 기반의 진단분류를 구축하는 데 목적을 두었다. 따라서 표현 언어 발달과 긴밀한 관련이 있는 수용 언어의 발달과정이나 문해력 발달과정에 대해서는 다루 고 있지 않다. 또한 $\mathrm{AAC}$ 의사소통 능력에 영향을 미치는 다른 영역에 대해서도 논의하고 있지 않다는 점도 주지할 필요가 있 다. 마지막으로 의사소통 및 언어발달을 마친 성인이 후천적 이 유로 $\mathrm{AAC}$ 를 필요로 하게 된 경우, 아동 발달을 기준으로 개발 한 본 진단분류체계가 적절하게 적용되지 않을 수 있다는 점을 밝힌다. 장애 유형 및 중증도에 따른 후천적 의사소통 장애의 진단분류에 대해서는 별도의 문헌고찰과 연구가 이루어져야 할 것이다. 후속연구에서는 본 연구에서 근거를 토대로 개발한 $\mathrm{LAAC}-\mathrm{D}$ 를 실제 $\mathrm{AAC}$ 를 필요로 하는 대상자에게 적용해보 고 타당성 및 보완사항을 구체적으로 살펴볼 필요가 있겠다.

중심 단어 : 보완대체의사소통·의사소통 수준·진단·언어적 능력.

\section{Ethical Statement}

N/A

\section{Acknowledgments}

N/A

\section{Declaration of Conflicting Interests}

There are no conflict of interests.

\section{Funding}

This work was supported by research fund of Chungnam National University (\#2018-0603-01). 


\section{Author Contributions}

Conceptualization: Sangeun Shin, HyunJu Park. Data curation: Sangeun Shin. Formal analysis: Sangeun Shin, HyunJu Park. Funding acquisition: Sangeun Shin. Investigation: Sangeun Shin, HyunJu Park. Methodology: Sangeun Shin. Project administration: Sangeun Shin. Validation: Sangeun Shin, HyunJu Park. Visualization: Sangeun Shin. Writing-original draft: Sangeun Shin. Writing-review \& editing: Sangeun Shin, HyunJu Park. Approval of final manuscript: all authors.

\section{ORCID iDs}

Sangeun Shin

https://orcid.org/0000-0003-0148-7829

Hyunju Park

\section{REFERENCES}

Banajee, M., DiCarlo, C., \& Buras-Stricklin, S. (2003). Core vocabulary determination for toddlers. Augmentative and Alternative Communication, 19(2), 67-73.

Bates, E. (1974). Acquisition of pragmatic competence. Journal of Child Language, 1(2), 277-281.

Bates, E., Camaioni, L., \& Volterra, V. (1975). The acquisition of performatives prior to speech. Merrill-Palmer Quarterly of Behavior and Development, 21(3), 205-226.

Beukelman, D., Jones, R., \& Rowan, M. (1989). Frequency of word usage by nondisabled peers in integrated preschool classrooms. Augmentative and Alternative Communication, 5(4), 243-248.

Beukelman, D. R. \& Mirenda, P. (2013). Augmentative and Alternative Communication: Supporting Children and Adults with Complex Communication Needs. Baltimore, MD: Paul H. Brookes.

Blockberger, S. \& Sutton, A. (2003). Toward linguistic competence: Language experiences and knowledge of children with extremely limited speech. In Light, J. C., Beukelman, D. R., \& Reichle J. (1st ed.). Communicative Competence for Individuals Who Use AAC: From Research to Effective Practice (pp. 63-106). Baltimore, MD: Paul H. Brookes.

Bloomberg, K., West, D., Johnson, H., \& Iacono, T. (2009). The Triple C: Checklist of Communication Competencies. Melbourne: Communication Resource Centre, Scope.

Brady, N., Fleming, K., Thiemann-Bourque, K., Olswang, L., Dowden, P., Saunders, M., et al. (2012). Development of the communication complexity scale. American Journal of Speech-Language Pathology, 21(1), 16-28.

Chadwick, D., Buell, S., \& Goldbart, J. (2019). Approaches to communication assessment with children and adults with profound intellectual and multiple disabilities. Journal of Applied Research in Intellectual Disabilities, 32(2), 336-358.

Darley, F. (1991). A philosophy of appraisal and diagnosis. In Darley, F. \& Spriestersbach, D. (2nd ed.). Diagnostic Methods in Speech Pathology (pp. 1-23). Prospect Heights, IL: Waveland Press.

DeLoache, J. S., Strauss, M. S., \& Maynard, J. (1979). Picture perception in infancy. Infant Behavior and Development, 2, 77-89.

Dodd, J. L. (2017). Augmentative and Alternative Communication Intervention: An Intensive, Immersive, Socially Based Service Delivery Model. (1st ed.). San Diego, CA: Plural Publishing.

Dodd, J. L. \& Gorey, M. (2014). AAC intervention as an immersion model. Communication Disorders Quarterly, 35(2), 103-107.

Dowden, P. A. (1999). Augmentative \& alternative communication for children with motor speech disorders. In Caruso, A. \& Strand, E. A. Clinical Management of Motor Speech Disorders of Children (pp. 345384). New York, NY: Thieme.

Fowler, A. E. (1998). Language in mental retardation: Associations with and dissociations from general cognition. In Burack, J. A., Hodapp, R. M., \& Zigler, E. Handbook of Mental Retardation and Development (pp. 290-333). Cambridge: Cambridge University Press.

Hanson, E. K., Beukelman, D. R., \& Yorkston, K. M. (2013). Communica-

tion support through multimodal supplementation: A scoping review. Augmentative and Alternative Communication, 29(4), 310-321.

Kim, Y. T. (2003). Assessment and Treatment of Language Disorders in Children. (1st ed.). Seoul: Hakjisa.

Kim, Y. T., Park, E. H., Han, S. K., \& Ku, J. A. (2016). Program of the Korean AAC Assessment (KAA) and Intervention (KAI). Seoul: Hakjisa.

Kovach, T. M. (2009). AACP: Augmentative and Alternative Communication Profile: A Continuum of Learning. Austin, TX: Pro-Ed.

Light, J. (1989). Toward a definition of communicative competence for individuals using augmentative and alternative communication systems. Augmentative and Alternative Communication, 5(2), 137-144.

Light, J. \& McNaughton, D. (2014). Communicative competence for individuals who require augmentative and alternative communication: $\mathrm{A}$ new definition for a new era of communication? Augmentative and Alternative Communication, 30(1), 1-18.

Light, J. C. (2003). Shattering the silence: Development of communicative competence by individuals who use AAC. In Light, J. C., Beukelman, D. R., \& Reichle, J. (1st ed.). Communicative Competence for Individuals Who Use AAC: From Research to Effective Practice (pp. 3-38). Baltimore, MD: Paul H. Brookes.

Mar, H. H. \& Sall, N. (1999a). Dimensions of Communication: Assessing the Communication Skills of Individuals with Disabilities. Paterson, NJ: St. Joseph's Children's Hospital.

Mar, H. H. \& Sall, N. (1999b). Profiles of the expressive communication skills of children and adolescents with severe cognitive disabilities. Education and Training in Mental Retardation and Developmental Disabilities, 34(1), 77-89.

Marvin, C., Beukelman, D., \& Bilyeu, D. (1994). Vocabulary-use patterns in preschool children: Effects of context and time sampling. Augmentative and Alternative Communication, 10(4), 224-236.

Mineo Mollica, B. (2003). Representational competence. In Light, J. C., Beukelman, D. R., \& Reichle, J. (1st ed.). Communicative Competence for Individuals Who Use AAC: From Research to Effective Practice (pp. 107146). Baltimore, MD: Paul H. Brookes.

Owens, R. E. (2015). Language Development: An Introduction. (9th ed.). Boston, MA: Pearson.

Peterson, H. A. \& Marquardt, T. P. (1994). Appraisal and Diagnosis of Speech and Language Disorders. (3rd ed.). Englewood Cliffs, NJ: Prentice Hall.

Rondal, J. (1994). Exceptional cases of language development in mental retardation: The relative autonomy of language as a cognitive system. In Tager-Flusberg, H. (1st ed.). Constraints on Language Acquisition: Studies of Atypical Children (pp. 155-174). Mahwah, NJ: Laurence Erlbaum Associates.

Rowland, C. (2016, June 24). The Communication Matrix. Retrieved from http://communicationMatrix.org.

Shin, S. E. \& Park, D. E. (2020). A literature review of AAC core vocabulary lists of children and adults. Journal of Speech-Language and Hearing Disorders, 29(1), 71-83.

Sigafoos, J., Woodyatt, G., Keen, D., Tait, K., Tucker, M., Roberts-Pennell, D., et al. (2000). Identifying potential communicative acts in children with developmental and physical disabilities. Communication Disorders Quarterly, 21(2), 77-86.

Smith, M. \& Grove, N. (1999). The bimodal situation of children learning language using manual and graphic signs. In Loncke, F. T., Clibbens, J., Arvidson, H. H., \& Lloyd, L. L. Augmentative and Alternative Communication: New Directions in Research and Practice (pp. 8-30). London: Whurr Publishers.

Smith, M. M. \& Grove, N. C. (2003). Asymmetry in input and output for individuals who use AAC. In Light, J. C., Beukelman, D. R., \& Reichle, J. (1st ed.). Communicative Competence for Individuals Who Use AAC: From Research to Effective Practice (pp. 163-195). Baltimore, MD: Paul H. Brookes.

Stephenson, J. \& Linfoot, K. (1996). Pictures as communication symbols for students with severe intellectual disability. Augmentative and Al- 
ternative Communication, 12(4), 244-256.

Vanderheiden, G. C. \& Yoder, D. E. (1986). Overview. In Blackstone, S. W. (1st ed.). Augmentative Communication: An Introduction (pp. 1-28). Rockville, MD: American Speech-Language-Hearing Association.

Visvader, P. (2013). AAC Basics and Implementation: How to Teach Students Who "Talk with Technology". Boulder, CO: Boulder Valley School District.
Volterra, V., Caselli, M. C., Capirci, O., \& Pizzuto, E. (2005). Gesture and the emergence and development of language. In Tomasello, M. \& Slobin, D. I. Beyond Nature-Nurture: Essays in Honor of Elizabeth Bates (pp. 3-41). Mahwah, NJ: Erlbaum.

Wetherby, A. M. \& Prizant, B. M. (1989). The expression of communicative intent: Assessment guidelines. Seminars in Speech and Language, 10(1), 77-91. 
APPENDIX

\section{언어 기반 AAC 진단분류체계[Language-Based AAC Diagnostic Classification System (LAAC-D)]}

\begin{tabular}{l|c|c|c}
\hline \multirow{2}{*}{ 의사소통 수준(communication levels) } & \multicolumn{3}{|c}{ 독립성 수준(independence level) } \\
\cline { 2 - 4 } & $\begin{array}{c}\text { 학습수준 } \\
\text { Learning (L) }\end{array}$ & $\begin{array}{c}\text { 출현수준 } \\
\text { Emerging (E) }\end{array}$ & $\begin{array}{c}\text { 습득수준 } \\
\text { Mastered (M) }\end{array}$ \\
\hline I. 전의도적 의사소통(preintentional communication) & (L) & (E) & (M) \\
II. 의도적 비상징 의사소통(intentional nonsymbolic communication) & (L) & (E) & (M) \\
III. 의도적 상징 의사소통(intentional symbolic communication)* & & (E) & (M) \\
III-1. 단단어 수준(single word level) & (L) & (E) & (M) \\
III-2. 단어 조합 수준(sord combination level) & (L) & (E) & (M) \\
III-3. 기본 문법 규칙을 사용한 단어 조합 수준(word combination level using \\
basic grammatical rules) (e.g., simple sentence forms using a few \\
$\begin{array}{l}\text { articles or morphemes) } \\
\text { III-4. 복잡한 문법 규칙을 사용한 언어 수준(language level using more }\end{array}$ \\
$\begin{array}{l}\text { complex grammatical rules) (e.g., compound and/or complex } \\
\text { sentence forms using various articles and morphemes) }\end{array}$ & (L) & (E) & (M) \\
\hline
\end{tabular}

*I단계에서는 말, 수신호, 몸짓, 그리고/또는 AAC 상징 선택이 단독으로 또는 함께 사용될 수 있음(speech, manual signs, gestures, and/or AAC symbol selection can be used alone or together in the stage III). AAC: augmentative and alternative communication 